\title{
Development of Compact Flexible Displacement Sensors Using Ultrasonic Sensor for Wearable Actuators
}

\author{
Tetsuya Akagi ${ }^{1, a}$, Shujiro Dohta ${ }^{1}$, Takafumi Morimoto ${ }^{1}$, Yasuko Matsui ${ }^{1}$ and So Shimooka ${ }^{1}$ \\ ${ }^{1}$ Department of Intelligent Mechanical Engineering, Okayama University of Science, Japan
}

\begin{abstract}
In position control of wearable actuator such as a rubber artificial muscle, a compact flexible displacement sensor is much attractive and required. In this paper, two types of flexible displacement sensor using the ultrasonic sensor were introduced. One is a built-in displacement sensor for rubber artificial muscle. Another is a sensor that can measure the sliding displacement on a flexible tube for flexible robot. Both sensors use ultrasonic displacement sensors. The construction, operating principle and measuring performance of two sensors were also described.
\end{abstract}

\section{Introduction}

Recently, pneumatic welfare systems to support a nursing care and a rehabilitation of the elderly and the disabled are actively researched and developed[1-4]. In the system, many wearable actuators such as a rubber artificial muscle have been used[5-7]. The systems using the wearable actuators had been sold in Japanese market. However, a position feedback control had not been embedded into the system because of lack of low-cost and compact flexible displacement sensor for these actuators. The target of this study is to develop a low-cost and compact flexible displacement sensor for a wearable actuator. In the previous study, the indirect displacement measurement using an inner diameter sensor of rubber artificial muscle had been proposed and tested[7-9]. To decrease the measuring error, the direct measurement is useful. In addition, the flexible robot using flexible pneumatic cylinders was proposed and tested in the previous study[10-11]. In the attitude control system of the robot arm, a compact flexible displacement sensor is also required. As a compact flexible displacement sensor, an ultrasonic sensor is selected because it has many advantages such as non-contacting measuring and relatively inexpensive.

\section{Built-in displacement sensor for rubber artificial muscle}

Figure 1 shows a schematic diagram of a tested measuring system of a rubber artificial muscle (Festo Co. Ltd., MXAM-20-AA, original length of $220 \mathrm{~mm}$ ) with a built-in sensor and an embedded controller (Renesas Co. Ltd. H8/3664). For applying the typical ultrasonic sensor on the market (Parallax Inc. Ltd., 28015) into the pressurized environment, the smaller transmitter (T4008A1) and receiver (R4008A1) produced by Nippon Ceramic Co. Ltd were replaced and installed into the rubber artificial muscle. The measuring procedure of the ultrasonic sensor using the embedded controller is

\footnotetext{
${ }^{\text {a }}$ Corresponding author : akagi@are.ous.ac.jp
} 
mentioned more detail in next section. The counting value corresponding to length of muscle is converted to the analogue signal in a $\mathrm{D} / \mathrm{A}$ converter on the measuring board.

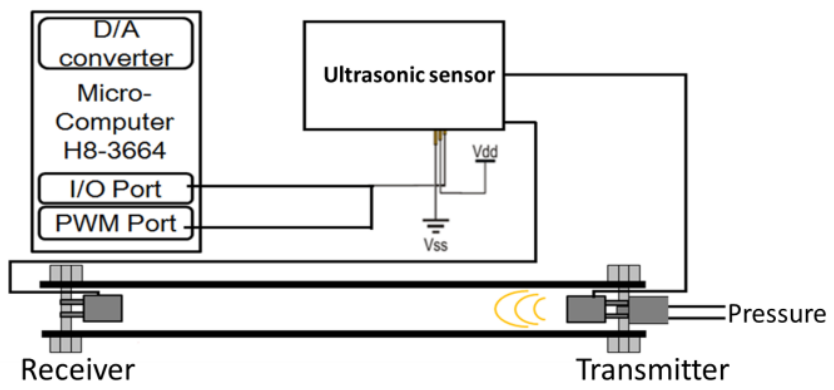

Figure 1. Tested measuring system of rubber artificial muscle.

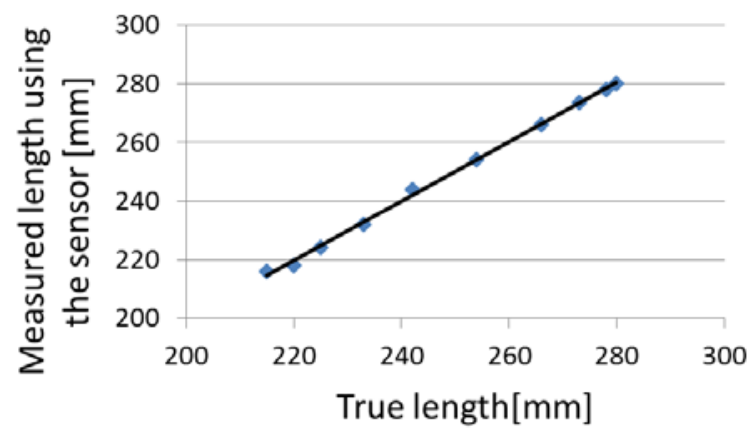

Figure 2. Comparison of measured and true length of the muscle.

Figure 2 shows the comparison between the measured length using tested sensor and the true length of the muscle measured by using a typical potentiometer on the market. In the experiment, the rubber artificial muscle was pressurized from $0 \mathrm{kPa}$ (the length of the muscle: $280 \mathrm{~mm}$ ) to $500 \mathrm{kPa}$ $(216 \mathrm{~mm})$. In Fig.2, the symbol shows the experimental result, the solid line shows the ideal case when the measured displacement is equal to true displacement. From Fig.2, it can be seen that the relation between the sensor output and the true length of the muscle is linear. This result means that the tested ultrasonic sensor is useful as a built-in displacement sensor of the muscle. The theoretical resolution calculated from the sampling period of counting and the speed of sound $(343.26 \mathrm{~m} / \mathrm{s}$ at 20 degree Celsius) of the sensor is about $0.2 \mathrm{~mm}$.

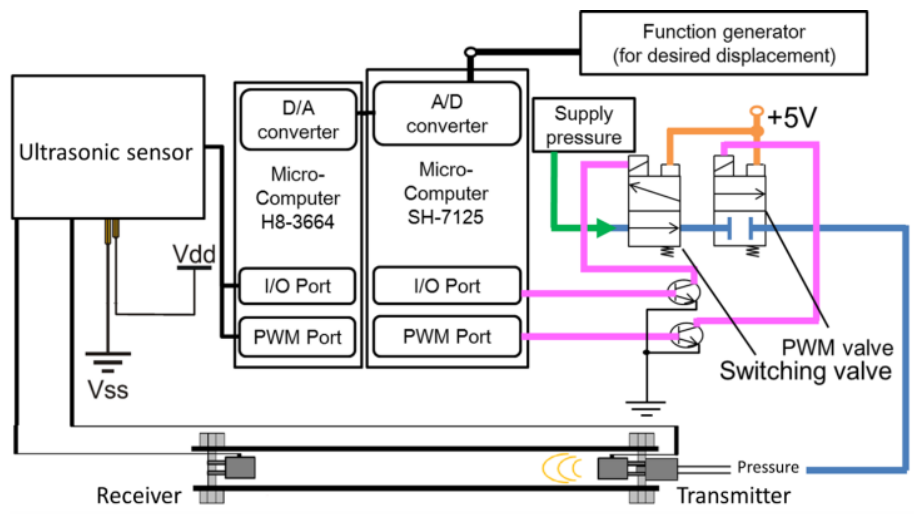

Figure 3. Positon control system using the rubber artificial muscle with a built-in displacement sensor. 
Figure 3 shows the position control system using the tested muscle with built-in displacement sensor. The position control system consists of the muscle with built-in displacement sensor, the tested measuring system as shown in Fig.1, a position controller using embedded controller (Renesas Co. Ltd., SH7125) and a quasi-servo valve that consist of PWM controlled and switching on/off valves[12]. The position control is done as follows. First, the embedded controller for the ultrasonic sensor counts the time of the output signal from the tested sensor through $\mathrm{I} / \mathrm{O}$ port. The embedded controller sends the measured displacement of the muscle length to another embedded controller as a voltage signal through the extra $\mathrm{D} / \mathrm{A}$ converter. The displacement deviation from the desired is calculated by the controller for positioning and the control input for the quasi-servo valve is also calculated based on P control scheme. Finally, the control input is applied to the quasi-servo valve as PWM signal and on/off signal. The sampling period of control is $15 \mathrm{~ms}$.

Figure 4 shows the transient response of displacement of the muscle controlled by using the tested system. In Fig.4, blue broken and red solid lines show the desired and controlled position, respectively. It can be seen that the position control can be realized by using the tested sensor.

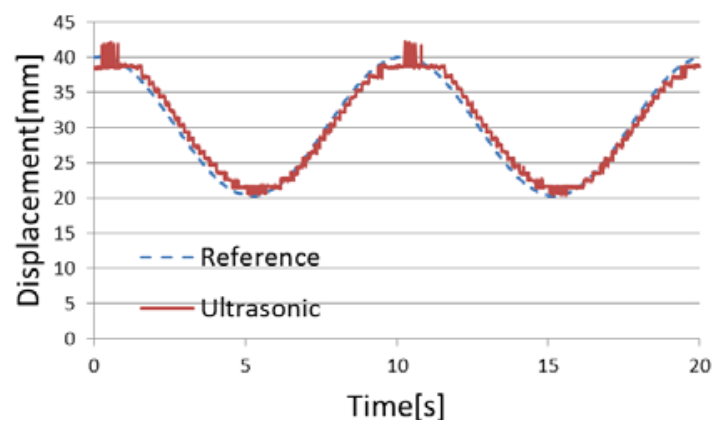

Figure 4. Transient response of displacement of the rubber artificial for positon tracking control.

\section{Flexible displacement sensor using flexible tube}

As an another sensor using ultrasonic sensor, the flexible sliding displacement sensor using flexible tube was proposed and tested. The sensor was developed to measure the displacement of the flexible robot arm for wrist rehabilitation device [11] as shown in Fig.5 on the left so as to construct the compact and low-cost measuring system with flexibility.
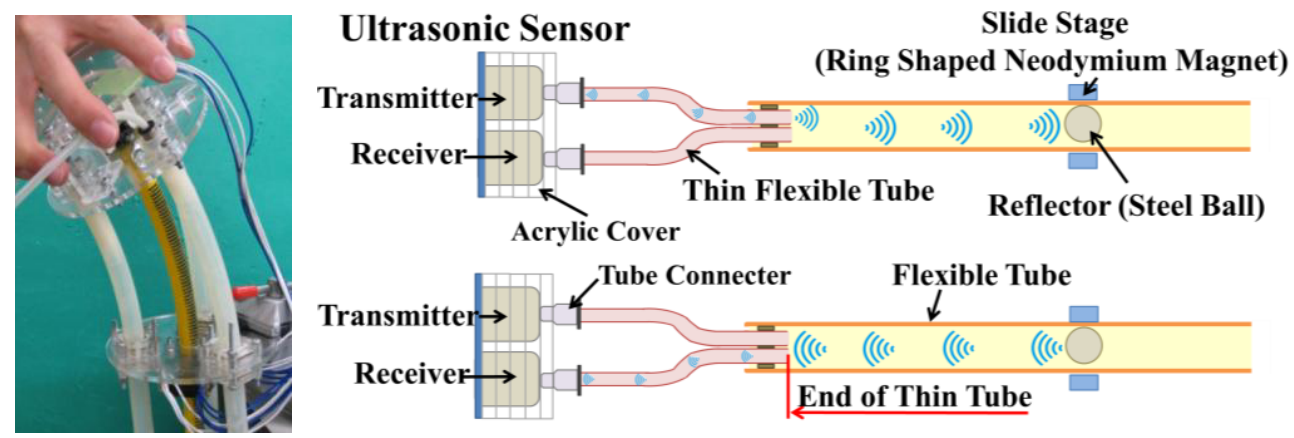

Figure 5. Flexible robot arm using flexible pneumatic cylinders and flexible sliding displacement sensor.

Figure 5 on the right shows the construction of the proposed sensor using same ultrasonic sensor. The tested sensor consists of the ultrasonic sensor mentioned above, an acrylic cover with two tube connecters and a thick flexible tube with an inner/outer diameter of $8 / 12 \mathrm{~mm}$ connected to two thin flexible tubes with an inner/outer diameter of $2.5 / 4 \mathrm{~mm}$. As a sliding stage, the ring-shaped neodymium magnet with an inner/outer diameter of $13 / 25 \mathrm{~mm}$, thickness of $5 \mathrm{~mm}$ and an acrylic cylindrical reflector with an outer diameter of $7.5 \mathrm{~mm}$ and length of $9 \mathrm{~mm}$ that a steel ball with an 
outer diameter of $5 \mathrm{~mm}$ were used. Figure 6 shows the construction and operating principle of the measuring system using the tested sensor. The measuring system consists of the tested sensor, the slide stage and an embedded controller (Renesas Co. Ltd., SH7125). I/O ports of the embedded controller are connected to the ultrasonic sensor to control the sensor. The cost of the tested sensor that includes the ultrasonic sensor and a magnet is low, that is about 35 U.S. dollars.

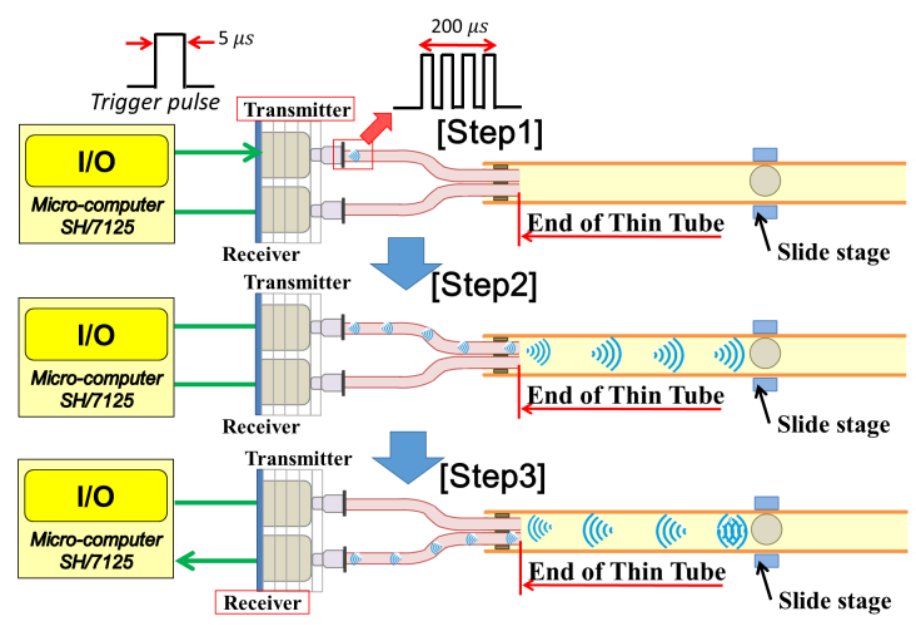

Figure 6. Construction and operating principle of measuring system using flexible sliding displacement sensor.

The measuring procedure is as follows. First, the embedded controller sends the trigger pulse for 5 $\mu$ s. Then, the transmitter is driven for $0.2 \mathrm{~ms}$ with frequency of $40 \mathrm{kHz}$ (Step 1 in Fig.6). The generated ultrasonic wave passes through the thin tube as a transmission tube. It reaches at the end of the tube, and is transmitted to the thick tube(Step 2 in Fig.6). After that, the ultrasonic wave reflects at the slide stage, and reaches at the ultrasonic receiver through another thin flexible tube (Step3 in Fig.6). The embedded controller can calculate the distance between the ultrasonic sensor and the slide stage by measuring the time (counting value) until the receiver detects the ultrasonic wave from the transmitter. By this method, the tested sensor can measure the displacement of slide stage even if the flexible tube bends.

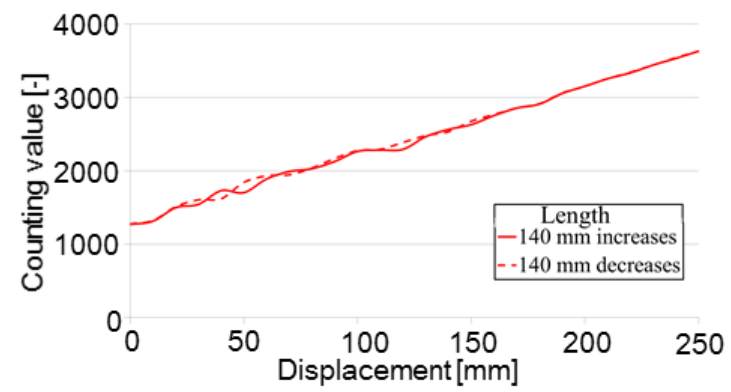

Figure 7. Relation between counting value and displacement.

Figure 7 shows the relation between the displacement of the slide stage and the counting value by using the thin tube with length of $140 \mathrm{~mm}$. In Fig.7, the solid and broken lines show the case that displacement of slide stage increases and decreases, respectively. It can be found that it has relatively good linear relationship. The displacement resolution of the sensor can be calculated from the counting sampling period of counting $(0.602 \mu \mathrm{s})$ and speed of sound $(343.26 \mathrm{~m} / \mathrm{s})$, that is about 0.1 $\mathrm{mm}$. The sampling period for measuring using the embedded controller is about $4 \mathrm{~ms}$ at in the case of measuring displacement of $0.2 \mathrm{~m}$. It is also found that it could measure the displacement even if the thin and thick tubes bends. 


\section{Conclusions}

Two types of flexible displacement sensor using the ultrasonic sensor and its measuring system using embedded controller were proposed and tested. The measuring performance of both measuring systems were investigated. As a result, it can be confirmed that the tested system could be applied to the positioning system of the rubber artificial muscle and the flexible robot arm using flexible pneumatic cylinder because of available resolution of 0.1 to $0.2 \mathrm{~mm}$ and relatively faster sampling period of 4 or $15 \mathrm{~ms}$ for rehabilitation device that requires slower motion.

\section{Acknowledgement}

This work was partially supported by the Ministry of Education, Culture, Sports, Science and Technology of Japan through a QOL Innovative Research Program (2012-) and Grant-in-Aid for Scientific Research (C) (Subject No. 24560315).

\section{References}

1. T. Noritsugu, M. Takaiwa, D. Sasaki, Development of Power Assist Wear Using Pneumatic Rubber Artificial Muscles, Journal of Robotics and Mechatronics, Vol. 21, 607-613(2009).

2. H. Kobayashi, T. Shiban, Y. Ishida, Realization of all 7 motions for the upper limb by a muscle suit, Journal of Robotics and Mechatronics, Vol. 16, 504-512(2004).

3. M. Ishii, K. Yamamoto, and K. Hyodo, Stand-Alone Wearable Power Suit -Development and Availability-, Journal of Robotics and Mechatronics, Vol. 17, No.5, 575-583(2005).

4. J. Piquion, A. Nayar, A. Ghazaryan, R. Papann, W. Klimek, and R. Laroia, Robot-assisted gynecological surgery in a community setting, Journal of Robotics and Surgery; Vol.3, Issue 2, 61-64(2009).

5. Y. Nagata, ed., Soft Actuators -Forefront of Development-, NTS Ltd., 291-335(2004).

6. T.Akagi, S.Dohta, Development of Wearable Pneumatic Actuator and Multiport Pressure Control Valve, Journal of Robotics and Mechatronics, Vol.17, No.5, 529-536(2005).

7. T. Akagi, S. Dohta, M.Ihara, Improvement of McKibben Artificial Muscle with Long Stroke Motion and Its Application, Journal of System Design and Dynamics, Vol.4, No.4, 538-551 (2010),

8. T.Akagi, S.Dohta, Y.Kenmotsu, F.Zhao, M.Yoneda, Development of smart inner diameter sensor for position control of Mckibben artificial muscle, Journal of Procedia Engineering, Vol.41, 105 $-112(2012)$.

9. T.Akagi, S.Dohta, Y.Kenmotsu, S.Jinno, K.Taniguchi, Model Based Axial Displacement Measurement of Rubber Artificial Muscle Using Inner Diameter Sensor, Lecture Notes in Electrical Engineering 293, Vol 1, Springer, 349-357(2014).

10. T. Morimoto, Mohd Aliff, T.Akagi, S.Dohta, Development of Flexible Pneumatic Cylinder with Backdrivability and Its Application, International Journal of Materials Science and Engineering, Vol.3, No.1, 7-11(2015).

11. Mohd Aliff, S. Dohta, T. Akagi, T. Moimoto, Control of Flexible Pneumatic Robot Arm Using Master Device with Pneumatic Brake Mechanism, JFPS International Journal of Fluid Power System, Vol.8, No.1, 38-43(2015).

12. Y. Moriwake, T.Akagi, S.Dohta, F.Zhao, Development of low-cost pressure control type quasiservo valve using embedded controller, Journal of Procedia Engineering, Vol.41, 143-149(2012). 